\title{
Clinical and Radiological Follow-Up Results of Patients with Sequestered Lumbar Disc Herniation: A Prospective Cohort Study
}

\author{
Hamza Sucuoğlua, b Abdullah Yüksel Barut ${ }^{a}$ \\ aSchool of Health Sciences, Istanbul Gelisim University, Istanbul, Turkey; ${ }^{\text {bDepartment }}$ of Physical Medicine and \\ Rehabilitation, Private Nisa Hospital, Istanbul, Turkey
}

\section{Significance of the Study}

- Spontaneous regression can be observed with conservative treatment in most patients with sequestered lumbar disc herniation (LDH).

- Although pain and disability scores were better in operated patients who underwent early surgery, they were similar to those in patients who regressed spontaneously at 6 months.

- We recommend conservative treatment in the early period in patients with sequestered LDH with no surgical indication.

\section{Keywords}

Lumbar disc herniation · Magnetic resonance imaging · Neurology · Pain · Radiology · Rehabilitation · Sequestered lumbar disc herniation $\cdot$ Spontaneous regression

\footnotetext{
Abstract

Purpose: The aim of the study was to assess radiological changes and clinical outcomes of patients with sequestered lumbar disc herniation (LDH) and evaluate the relationship between them. Methods: Patients diagnosed with sequestered LDH were followed up in 2 groups: operated (within the 1st month after diagnosis) and nonoperated. Visual analog scale (VAS) and Oswestry Disability Index (ODI) scores at baseline (V1) and 1st (V2), 3rd (V3), and 6th (V4) month visits were used for clinical evaluation. Radiological evaluation was performed by measuring the sequestered LDH level and herniation volume using magnetic resonance imaging (MRI) at V1 and V4. After the second MRI, patients in the nonoper-
}

ated group were divided into 3 groups: nonregression ( $n=$ $6)$, partial regression $(n=22)$, and complete resolution ( $n=$ $27)$; patients were analyzed in 4 groups including the ones in the operated ( $n=25$ ) group. Results: Significant improvements were observed in VAS and ODI scores at V2 and V3 in all groups $(p=0.000)$ and at $\mathrm{V} 4$ in partial regression and complete resolution groups $(p=0.000)$. VAS and ODI score improvements at V2 and V3 were significantly higher in the operated group than in other groups $(p=0.000)$. At V4, there were no significant differences in VAS and ODI scores $(p>$ 0.05 ) between the operated group and partial regression and complete resolution groups. Conclusion: Spontaneous regression was observed in the 6th month post-MRI in most of the nonoperated sequestered LDH patients with conservative treatment. Improvements in pain and disability scores were higher among the operated patients at the early stage, whereas they were not significantly different compared to patients with spontaneous regression at the 6th month.

(C) 2021 The Author(s)

Published by S. Karger AG, Basel karger@karger.com www.karger.com/mpp

Karger $\stackrel{\text { ' }}{5}$

BOPEN ACCESS
(C) 2021 The Author(s)

Published by S. Karger AG, Basel

This is an Open Access article licensed under the Creative Commons Attribution-NonCommercial-4.0 International License (CC BY-NC) (http://www.karger.com/Services/OpenAccessLicense), applicable to the online version of the article only. Usage and distribution for commercial purposes requires written permission. 


\section{Introduction}

Lumbar disc herniation (LDH) causes lower back pain and radicular pain and functional constraints, disrupting the quality of life. LDH has 5 identified herniation subtypes: bulging discs (mildest form), focal protrusions, broad-based protrusions, extrusions, and sequestrations (the most severe form) $[1,2]$. The distinction between sequestered LDH and other hernia subtypes showing similar clinical findings can be demonstrated using magnetic resonance imaging (MRI) [3]. Sequestrations, also known as free fragments, are fragments of nucleus pulposus and annulus fibrosus separated from the intervertebral discs $[2,4]$. Among the LDH subtypes, the prevalence of sequestrations is $1.7-3 \%[5,6]$; however, the prevalence of surgery is 7\% [5]. The concern that neurological deficits may develop in patients with sequestered LDH may increase the rate of preference for surgical intervention [7]. However, spontaneous regression of LDH has long been well known [8-10]. In general, larger herniations are known to regress faster than smaller ones, and the greatest regression is observed in the sequestered subtype [911]. Moreover, successful results can be achieved with conservative treatment in most cases of sequestered LDH without absolute indications for surgery [9-11]. Determining the relationship between spontaneous regression and the improvement of clinical symptoms was done by following disc herniation and morphological changes in discs with serial MRI $[3,12]$.

A few case reports and reviews have reported spontaneous regression in sequestered $\mathrm{LDH}$ [9-11]. To the best of our knowledge, there is only one prospective cohort follow-up study [13]. Studies on clinical and radiological comparison of sequestered LDH patients who underwent surgery with those who underwent conservative treatment without surgery and their spontaneous regression images have not been reported. Thus, in this prospective cohort study, we aimed to detect the clinical outcomes and radiological changes in MRI (regression, resolution, or none) by performing a periodic follow-up of patients with operated and nonoperated sequestered LDH and to determine the relationship between them.

\section{Materials and Methods}

\section{Study Design}

We used a prospective cohort study design in accordance with the STROBE statement. The study protocol was approved by the Ethics Committee of Istanbul Gelisim University (Decision No. 19.04-20/05.08-17). The study was conducted in accordance with the rules of the Declaration of Helsinki, and all individuals gave written consent prior to participation.

\section{Participants}

This study was conducted at the Physical Therapy and Rehabilitation Clinic, Private Nisa Hospital, between October 2017 and April 2020. We included patients aged between 18 and 70 years who were admitted to the outpatient clinic with lower back pain and a sequestered LDH in the lumbar MRI scan. The patients' symptoms and physical examination findings were consistent with sequestered LDH. Sequestered LDH was diagnosed by detecting at least one plane herniated disc mass (fragment) separated from the disc. The exclusion criteria were as follows: pregnancy, spondylolisthesis, spinal stenosis, lower back pain combined with inflammatory diseases, vertebral fracture, spine infection and tumors, cauda equina syndrome, and a history of lumbar surgery.

Some of the patients diagnosed with sequestered LDH underwent surgery (within the 1st month after diagnosis), whereas most of them did not. Approximately 50\% of the patients who underwent surgery had muscle weakness, and some others had severe pain. The patients were divided into 2 groups at the first stage: those who underwent surgery (operated) and those who did not (nonoperated). After the control MRI taken at the 6th month, nonoperated patients were divided into 3 groups of nonregression, partial regression, and complete resolution and the 4th group of operated patients (those who underwent surgery at the first stage; Fig. 1). Nonoperated patients continued conservative treatment (e.g., analgesic or nonsteroidal anti-inflammatory drugs, myorelaxant drugs, physical therapy, exercises, lumbosacral orthosis, rest, and epidural steroids). These treatments were continued for up to 2 months for some patients; most of the patients received multiple treatments.

\section{Evaluations}

During the initial examination of the patients, the following parameters were recorded: age, sex, BMI, duration and location of pain, and muscle weakness. Clinical evaluation was conducted using the visual analog scale (VAS) [14] and the Turkish-approved version of the Oswestry Disability Index (ODI) [15] via face-toface interviews with the patients at baseline (V1) and 1st (V2), 3rd (V3), and 6th (V4) month visits. The evaluator was an experienced, independent physiatrist blinded to the radiological information. MRIs taken at V1 and V4 were used for radiological evaluations.

Visual Analog ScaleThe VAS assessment, which was used in this study to measure the patients' levels of pain, was made using figures from " 0 " to " 10 " marked equally in a $10-\mathrm{cm}$ line. The authors explained to the patients that " 0 " means no pain, " 5 " means moderate pain, and " 10 " means unbearable pain; the patients were asked to mark the appropriate score on the line that best described their pain [14].

\section{ODI Score}

The ODI is recognized as the "gold standard" of lumbar functional outcome instruments and focuses on physical activities. It consists of 10 physical activity sections rated from 0 to 5 . The total score is calculated by adding up all the points marked in each section. The total possible score is calculated by multiplying the number of marked episodes by 5 , and the maximum possible score is 50. The ODI total score (\%) is calculated by dividing the total score by the total possible score and multiplying the quotient by 100 . 


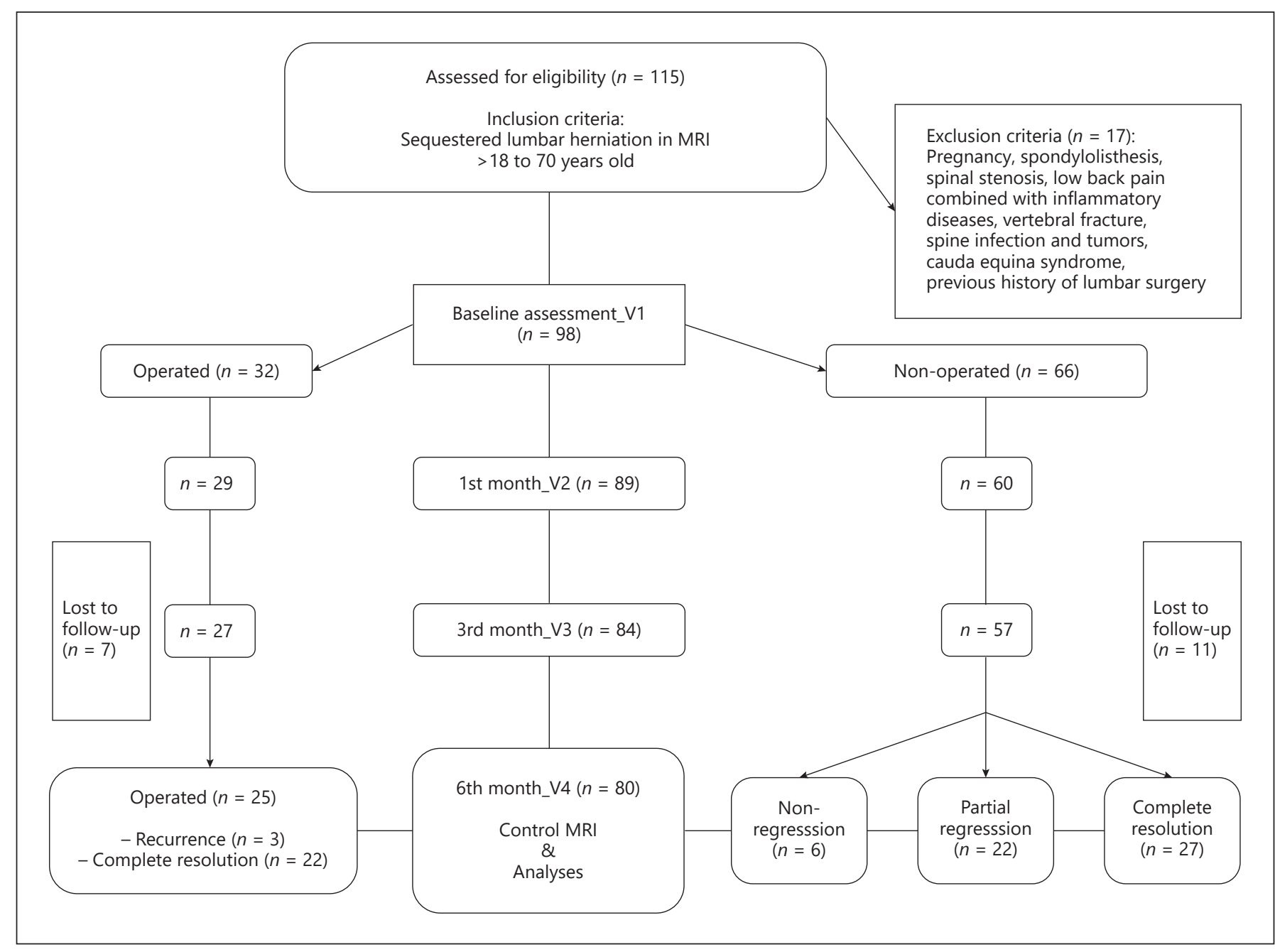

Fig. 1. Flow diagram of the study. MRI, magnetic resonance imaging.

High scores indicate that the individual is more affected by the disease [16].

\section{Magnetic Resonance Imaging}

All patients were imaged using a $1.5 \mathrm{~T}$ MRI scanner (Signa HDxt 1.5T; GE Healthcare, USA) with the spine coil in a supine position. Lumbar spinal MRI consisted of sagittal T1W, sagittal $\mathrm{T} 2 \mathrm{~W}$, and axial T2W images. The same experienced radiologist blinded to the clinical information evaluated MRI scans taken with the same device. The sequestered LDH level and sequestered hernia (fragment) volume of the patients were determined. The hernia volume $\left(\mathrm{mm}^{3}\right)$ was calculated by measuring the area $\left(\mathrm{mm}^{2}\right)$ in each axial image and multiplying this value by the scanning thickness $(4 \mathrm{~mm})$ plus intraslice clearance $(1 \mathrm{~mm})$ [7]. The patients were divided into 3 groups depending on the change in the volume of the sequestered disc hernia in the sagittal and axial views: (1) nonregression: no change in disk volume; (2) partial regression: $>25 \%$ herniation size reduction; and (3) complete resolution: herniated disc volume is absent (Fig. 2). In addition, recurrence was defined as recurring pain in operated patients along with the observation of disc hernia at the same level in the control MRI.

\section{Statistical Analyses}

All statistical analyses were performed using SPSS version 20.0 (IBM Corp., Armonk, NY, USA). Data were presented as mean \pm standard deviation (median [minimum-maximum]) for continuous variables, and as frequencies and percentages for categorical variables. Normality of the data was assessed using the ShapiroWilk test. The one-way ANOVA test was used for the intergroup comparison of continuous variables with normal distribution. The Wilcoxon signed-rank test was used for the intragroup comparison of continuous variables without normal distribution; the Kruskal-Wallis test was used for the cross-group comparison. When statistically significant differences were found between the groups, the Mann-Whitney U test was used to determine groups causing the difference. A $\chi^{2}$ test was used to compare the categorical variables. For all analyses, the level of significance was set at $p<0.05$. 
Fig. 2. View of caudal migrated sequestered disc hernia compressing the right lateral L5 root at the L4-L5 level on sagittal (a) and axial (b) sections in the MRI performed at V1 (baseline). View of complete resolution at the L4-L5 level on sagittal (c) and axial (d) sections in the MRI performed at V4 (follow-up). MRI, magnetic resonance imaging.
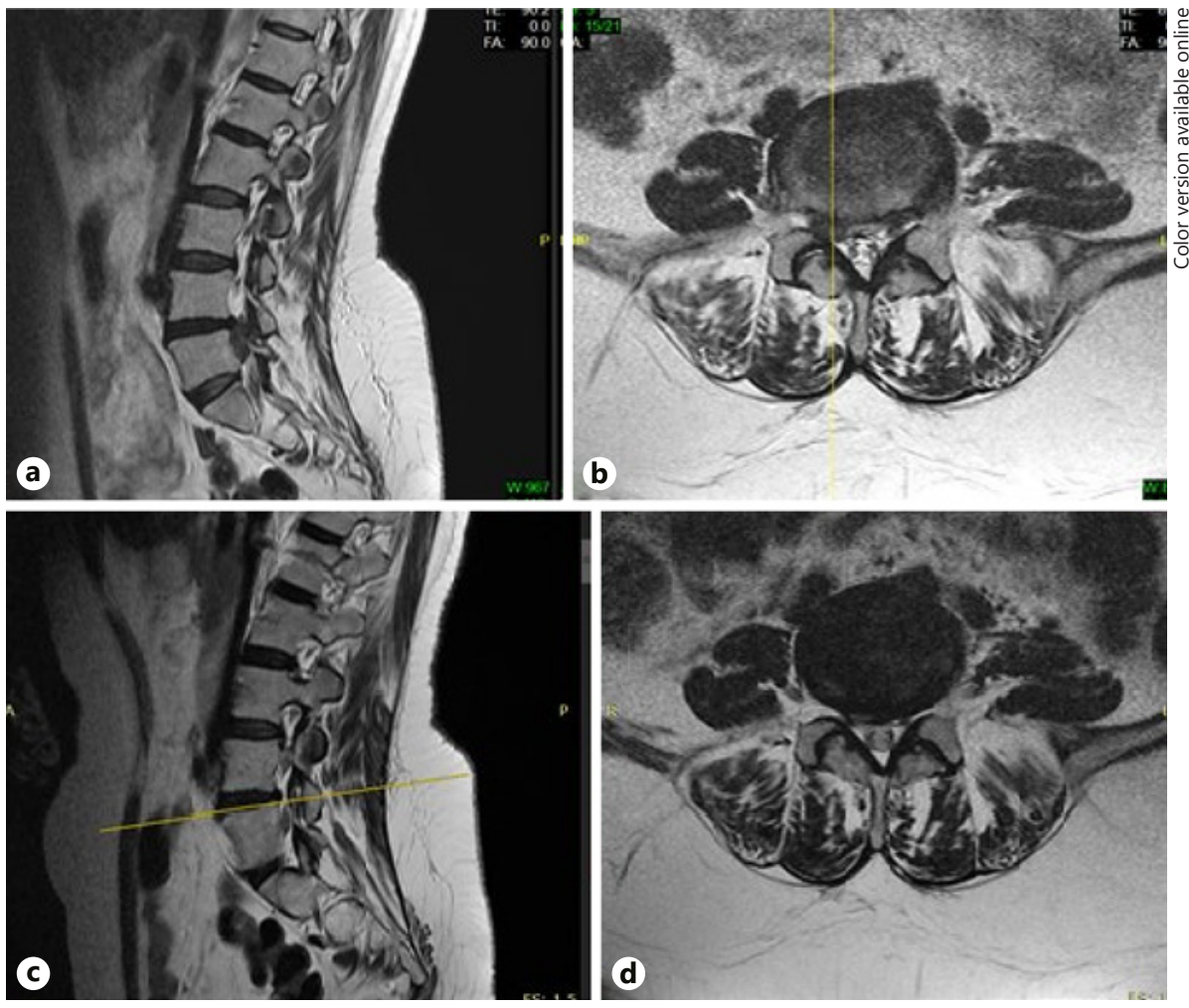

\section{Results}

A total of 115 sequestered LDH patients were evaluated, and 98 patients were finally included in the study. Of these patients, 32 (32.7\%) underwent surgery (operated), whereas 66 (67.3\%) did not undergo surgery (nonoperated) and received conservative treatment. Following the control MRI scan conducted in 80 patients with continuing follow-ups at V4, $6(10.9 \%)$ patients were included in the nonregression, $22(40 \%)$ in the partial regression, and $27(49.1 \%)$ in the complete resolution groups; 25 patients remained in the operated group. Statistical analysis was conducted with the 80 patients in these 4 groups (Fig. 1).

Table 1 shows the clinical and demographic characteristics of the patients. There was no significant difference between the groups in terms of mean age and duration of pain $(p>0.05)$. The sequestered LDH level was most commonly L4-L5 (37.5\%) and L5-S1 (37.5\%), with no significant difference between groups $(p>0.05)$. Muscle weakness was markedly more prevalent in the operated group at V1 $(48 \% ; p=0.003)$. In addition, the improvement in muscle weakness was statistically significant in the operated group $(p=0.001)$.

Clinical and Radiological Results of Sequestered Lumbar Disc Herniation
None of the nonoperated patients with continued V4 underwent surgery; recurrence was observed in 3 (12\%) of the operated patients. The duration from diagnosis to surgery among operated patients [mean \pm SD (median [minimum-maximum])] was $10.8 \pm 10.7(7.0[0-30])$ days.

There was no significant difference in the VAS and ODI scores between the groups at V1 $(p=0.710$ and $p=$ 0.954 , respectively) (Fig. 3,4 ). The nonregression group had significantly higher VAS and ODI scores at V2, V3, and V4 than the other groups $(p=0.000)$ (Fig. 3, 4). Significant improvements were observed in VAS scores at V2 and V3 in all groups $(p=0.000)$. Significant improvements in the VAS scores within the groups were continued in all follow-ups (V2, V3, and V4) in the partial regression and complete resolution groups $(p=0.000)$. However, VAS scores in the nonregression group and the operated group did not show a significant difference at V4 compared with V3 ( $p=0.083$ and $p=0.670$, respectively). The operated group had significantly lower VAS scores at V2 and V3 than the other groups $(p=0.000)$. At $\mathrm{V} 4$, the VAS scores in the operated group were no different from those of partial regression and complete resolution groups ( $p=0.812)$ (Fig. 3).

Med Princ Pract 2021;30:244-252 


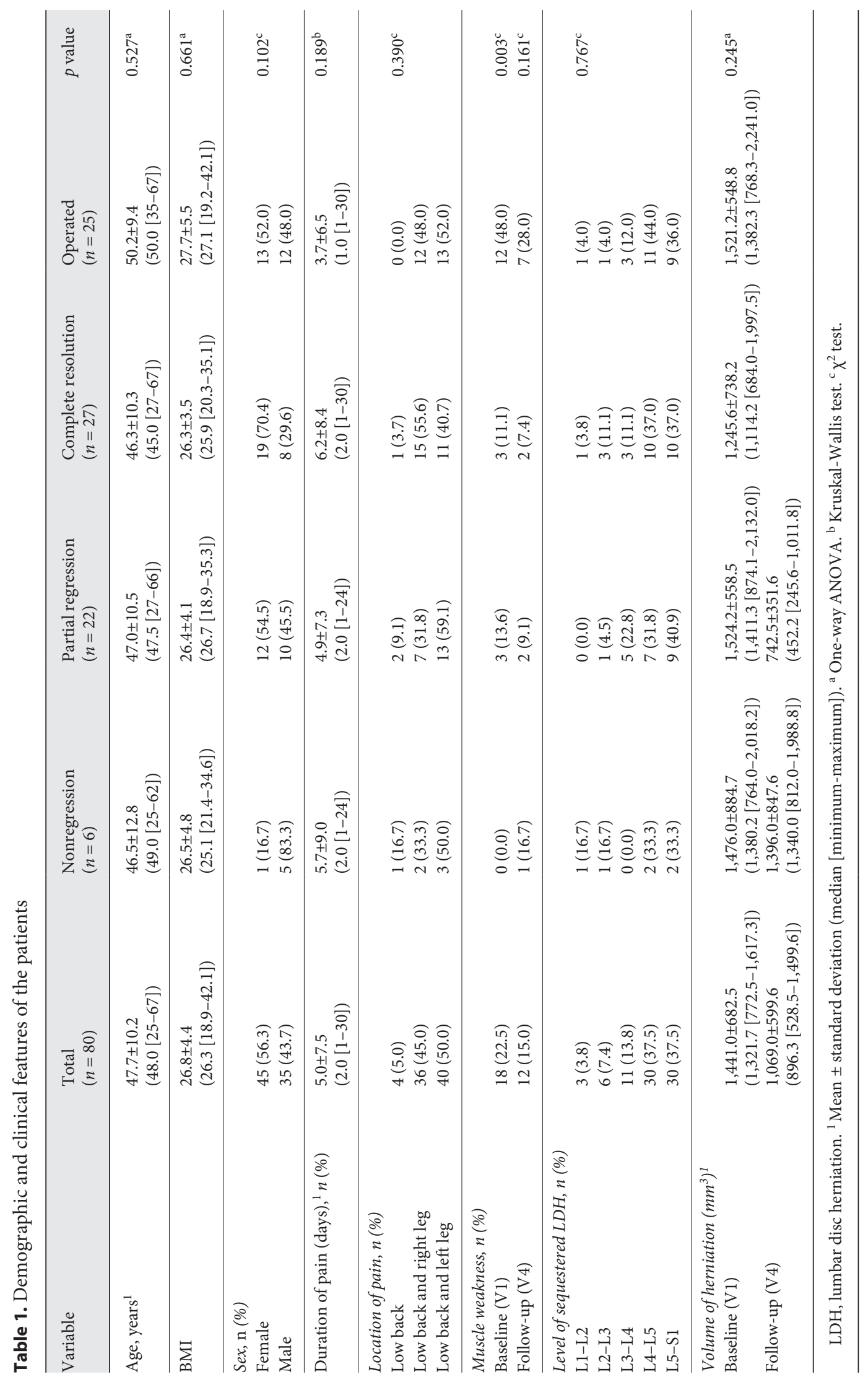




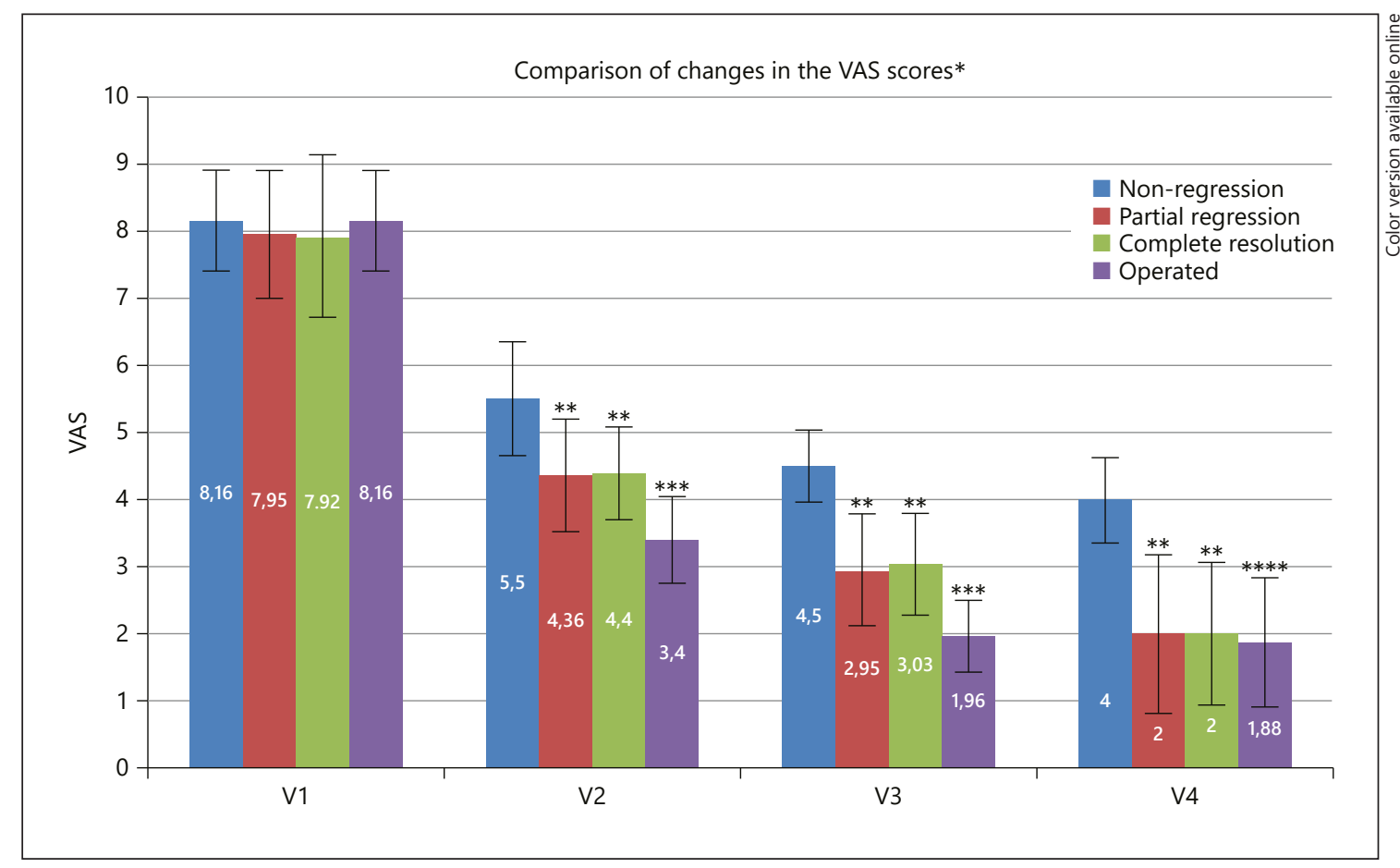

Fig. 3. Comparison of changes in the VAS scores over time between the groups. Lower values correspond to clinical improvement. Visits: baseline (V1); 1 st month (V2); 3rd month (V3); 6th month (V4). * Mean. ** Significant improvements in the VAS scores within the groups continued in all follow-ups (V2, V3, and V4) of partial regression and complete resolution groups $(p=0.000)$. ${ }^{* * *}$ The operated group had significantly lower VAS scores at V2 and V3 than the other groups $(p=0.000)$. ${ }^{* * * *}$ At V4, the VAS scores in the operated group were no different from those of partial regression and complete resolution groups $(p=0.812)$. VAS, visual analog scale.

Significant improvements in the ODI scores within the groups continued in all groups during V2, V3, and V4 $(p<0.05)$. The operated group had significantly lower ODI scores at V2 and V3 than the other groups $(p=$ $0.000)$. At V4, there was no significant difference in the ODI scores between the partial regression and complete resolution groups and the operated group $(p=0.800)$ (Fig. 4).

Table 1 shows the sequestered disc herniation volumes of all groups at V1 and V4. It can be observed that there was no significant volume change in the nonregression group, whereas the sequestered disc volume at V4 was 0 $\mathrm{mm}^{3}$ in the complete resolution and operated groups. The average herniated disk volume (mean \pm SD) in the partial regression group decreased from 1,524.2 \pm 558.5 to $742.5 \pm 351.6 \mathrm{~mm}^{3}$.

\section{Discussion}

In this study, we present the clinical and radiological follow-up results of operated and nonoperated patients with sequestered LDH. Approximately $90 \%$ of patients with $\mathrm{LDH}$ are reported to have achieved good or perfect results with conservative treatment [17]. Spontaneous regression of disc herniation is a known phenomenon, and regression is more prevalent among sequestered disc hernias $[9,10]$. In this study, one-third of the patients underwent operation, and most of them had muscle weakness or severe pain. Clinical and radiological improvement was observed in approximately $90 \%$ of nonoperated patients with sequestered LDH who received conservative treatment.

Although the mechanism of spontaneous regression of disc herniation is not clearly understood, 3 hypotheses have been mentioned in the literature: first, the herniation could retract back into its main disc, which possibly occurs in disc bulging and disc protrusions [18]; second, 


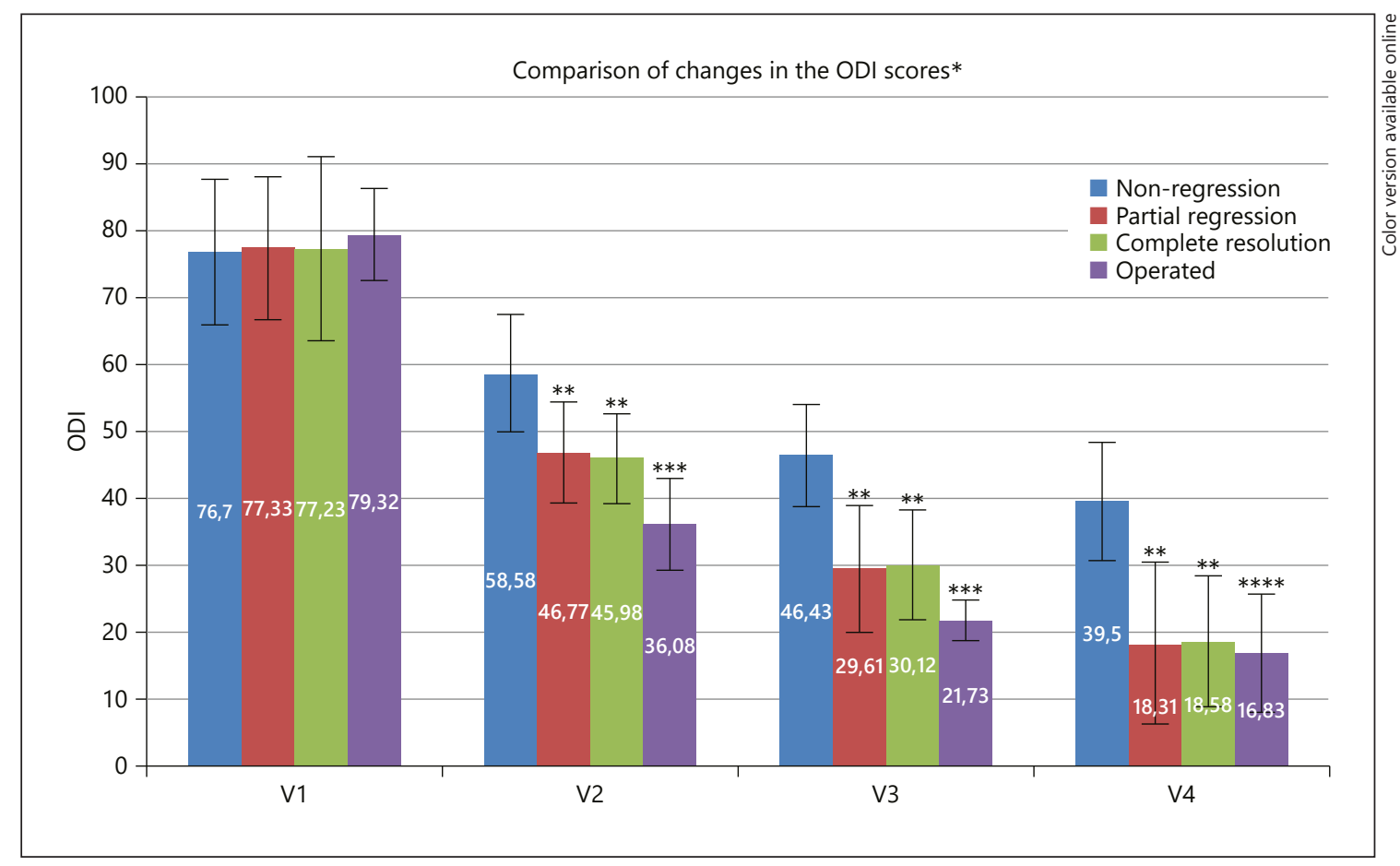

Fig. 4. Comparison of changes in the ODI scores over time between the groups. Lower values correspond to clinical improvement. Visits: baseline (V1); 1st month (V2); 3rd month (V3); 6th month (V4). * Mean. ** Significant improvements in the ODI scores within the groups continued in all follow-ups (V2, V3, and V4) of partial regression and complete resolution groups $(p<0.05)$. *** The operated group had significantly lower ODI scores at V2 and V3 than the other groups $(p=0.000)$. ${ }^{* * * *}$ At V4, the VAS scores in the operated group were no different from those of partial regression and complete resolution groups $(p=0.800)$. ODI, Oswestry Disability Index; VAS, visual analog scale.

dehydration of the hernia [19]; and third, herniation causing an inflammatory reaction and neovascularization in the epidural cavity and dissolving with macrophage phagocytosis and enzymatic degradation [10]. The third hypothesis has been described as being more persuasive than the other 2 hypotheses for spontaneous regression $[10,20]$. While dehydration contributes to a reduction in the size of a herniated disc, it cannot adequately explain the cases of complete regression of a free fragment [21]. The mechanism mediated by inflammation may have a role in spontaneous regression because the free fragment is more exposed to the peripheral circulation in the epidural space than in other types of hernia $[19,22]$, resulting in immunological reactions [22].

Spontaneous regression rates in LDH differ based on differences in experimental approaches and imaging methods, follow-up durations, and classification of disc herniation [7]. The spontaneous regression rates are higher among patients with disc sequestration (96\%) and extrusion (70\%) than among patients with protrusion (41\%) and disc bulging (13\%); moreover, disk sequestration has a much higher complete resolution rate (43\%) [10], suggesting that sequestered-type herniation is a predictive factor for spontaneous regression. Takada et al. [23] reported regression ( $>50 \%$ herniation size reduction) in $100 \%$ and complete resolution in $44 \%$ of 18 sequestered LDH cases, as observed in the MRI scans taken after 9 months. Macki et al. [11] studied 54 patients with sequestered $\mathrm{LDH}$ and found that spontaneous regression occurred within an average of 9 months and that regression probability was the highest among the sequestered type compared with the other hernia subtypes. Ahn et al. [3] reported regression ( $>25 \%$ herniation size reduction) in $100 \%$ and complete resolution in $64 \%$ of 11 sequestered $\mathrm{LDH}$ patients after an average follow-up period of 4.3 months. In our study, regression was observed in $89 \%$ and complete resolution in $49 \%$ of the patients in the nonoperated group in the MRI scans taken at the 6th month. 
While the regression rates in this study are close to those reported in the literature, these rates may increase with longer follow-up durations.

The relationship between spontaneous regression of disc hernia and improvement of clinical outcomes is controversial $[7,10]$. In addition to disc herniation, many physical and psychological factors of patients that may affect clinical results may be the cause of this inconsistency [10]. Disc regression was not the only factor to be associated with clinical improvement; the clinical outcome also showed improvement without regression [24]. In addition, the definition of regression differs between studies; studies have defined spontaneous regression as a reduction in the size of the disc hernia by $>20 \%$ [25], 50\% [23], or 70\% [12]. This makes it difficult to establish a relationship between clinical outcomes and regression. Despite all this, complete resolution, significant high regression rate, and rapid regression can be counted as factors for spontaneous disc regression that have better correlation with clinical improvement $[10,12,23]$. Among the 8 sequestered LDH patients in Takada et al.'s study [23] and 75 sequestered LDH patients of Rahimizadeh and Saghri's study [13] with complete resolution observed, all had good or excellent clinical results. Bazzao et al. [12] and Ahn et al. [25] found that a decrease in the size of the hernia by $>70$ and $>20 \%$, respectively, was associated with clinical improvement. In the current study, significant improvements in pain and disability scores were observed with a decrease in the hernia size of $>25 \%$. There was no difference in terms of clinical recovery between partial regression and complete resolution. At the 6th month, patients with spontaneous regression became clinically similar to operated patients.

The incidence rate of neurological deficit (66.7\%) [6] in sequestered LDH being higher than other hernia subtypes may increase the prevalence of surgery. Despite this, every surgical intervention without indication brings the risk of complications and relapses. Recurrence of up to $8 \%$ and a complication rate of up to $9 \%$ after discectomy have been reported [26]. Therefore, conservative treatment is recommended for at least 2 months in $\mathrm{LDH}$ patients with no absolute surgical indication, and surgery is suggested to be considered if conservative treatment is not successful [27]. In our study, the number of patients with muscle weakness who underwent surgery was high as could be predicted, and significant improvements were observed in motor deficit postoperatively. The prevalence of complications in operated patients was not evaluated, but the recurrence rate (12\%) was slightly higher than that in the literature.

Clinical and Radiological Results of Sequestered Lumbar Disc Herniation
In studies, comparing surgical and conservative treatment in LDH treatment, it was shown that pain reduced faster in the short term with surgery, but in medium- and long-term follow-ups, both treatments have similar benefits [28]. In this study, although the improvement in pain and disability scores for sequestered $\mathrm{LDH}$ patients was higher among the operated patients, they were found to be similar to those in patients with spontaneous regression at the 6 th month.

\section{Limitations of the Study}

The primary limitation of this study was that the follow-up duration was $<1$ year, despite having a standard imaging time for control MRI. Therefore, the study is far from showing long-term results. Second, the number of patients lost during the follow-up was relatively high. This limitation may have affected clinical and radiological results. Third, there is a lack of a standard definition in the literature for spontaneous regression. Fourth, as the nonoperated patients received various conservative treatments, the results do not show the effect of any single treatment.

\section{Conclusion}

Partial regression or complete resolution was observed with conservative treatment in the 6th month post-MRI in most of the nonoperated patients with sequestered LDH. Improvements in pain and disability scores were higher among the operated patients at the early stage, whereas they were found to be no different in patients with spontaneous regression at the 6th month. Thus, we recommend that the conservative treatment be attempted before surgery in patients with sequestered $\mathrm{LDH}$ without absolute surgical indication or uncontrollable pain.

\section{Statement of Ethics}

The study protocol was approved by the Ethics Committee of Istanbul Gelisim University (Decision No: 19.04-20/05.08-17). The study was conducted in accordance with the rules of the Declaration of Helsinki, and all individuals gave written consent prior to participation.

\section{Conflict of Interest Statement}

The authors declare that they have no conflict of interest. 


\section{Funding Sources}

This research did not receive any specific grant from funding agencies in the public, commercial, or not-for-profit sectors.

\section{Author Contributions}

H.S.: conceptualization, methodology, writing - review and editing, data curation, and formal analysis; A.Y.B.: review and editing, data curation, and formal analysis.

\section{References}

1 Fardon DF, Milette PC. Nomenclature and classification of lumbar disc pathology. Recommendations of the Combined Task Forces of the North American Spine Society, American Society of Spine Radiology, and American Society of Neuroradiology. Spine. 2001;26(5): E93-113.

2 Albert HB, Manniche C. The efficacy of systematic active conservative treatment for patients with severe sciatica: a single-blind, randomized, clinical, controlled trial. Spine. 2012;37(7):531-42.

3 Ahn SH, Park HW, Byun WM, Ahn MW, Bae $\mathrm{JH}$, Jang SH, et al. Comparison of clinical outcomes and natural morphologic changes between sequestered and large central extruded disc herniations. Yonsei Med J. 2002;43(3): 283-90.

4 Bajpai J, Saini S, Singh R. Clinical correlation of magnetic resonance imaging with symptom complex in prolapsed intervertebral disc disease: a cross-sectional double blind analysis. J Craniovertebr Junction Spine. 2013;4(1) $16-20$

5 Weinstein JN, Lurie JD, Tosteson TD, Skinner JS, Hanscom B, Tosteson AN, et al. Surgical vs nonoperative treatment for lumbar disk herniation: the Spine Patient Outcomes Research Trial (SPORT) observational cohort. JAMA. 2006;296(20):2451-9.

6 Lachman D. Analysis of the clinical picture in patients with osteoarthritis of the spine depending on the type and severity of lesions on magnetic resonance imaging. Reumatologia. 2015;53(4):186-91.

7 Kesikburun B, Eksioglu E, Turan A, Adiguzel E, Kesikburun S, Cakci A. Spontaneous regression of extruded lumbar disc herniation: correlation with clinical outcome. Pak J Med Sci. 2019 Jul-Aug;35(4):974-80.

8 Cribb GL, Jaffray DC, Cassar-Pullicino VN. Observations on the natural history of massive lumbar disc herniation. J Bone Joint Surg Br. 2007;89(6):782-4.
9 Zhong M, Liu JT, Jiang H, Mi W, Yu P-FCL, Xue RR. Incidence of spontaneous resorption of lumbar disc herniation: a meta-analysis. Pain Physician. 2017;6:45-52.

10 Chiu CC, Chuang TY, Chang KH, Wu CH, Lin PW, Hsu WY. The probability of spontaneous regression of lumbar herniated disc: a systematic review. Clin Rehabil. 2015;29(2): 184-95.

11 Macki M, Hernandez-Hermann M, Bydon M, Gokaslan A, McGovern K, Bydon A. Spontaneous regression of sequestrated lumbar disc herniations: literature review. Clin Neurol Neurosurg. 2014;120:136-41.

12 Bozzao A, Gallucci M, Masciocchi C, Aprile I, Barile A, Passariello R. Lumbar disk herniation: MR imaging assessment of natural history in patients treated without surgery. Radiology. 1992;185(1):135-41.

13 Rahimizadeh A, Saghri M. Spontaneous resolution of sequestrated lumbar disc herniation: a prospective cohort study. Global Spine J. 2016;6(Suppl 1):s-0036.)

14 Dixon JS, Bird HA. Reproducibility along a 10 $\mathrm{cm}$ vertical visual analogue scale. Ann Rheum Dis. 1981;40(1):87-9.

15 Yakut E, Düger T, Oksüz C, Yörükan S, Ureten $\mathrm{K}$, Turan D, et al. Validation of the Turkish version of the Oswestry Disability Index for patients with low back pain. Spine. 2004; 29(5):581-5.

16 Fairbank JC, Pynsent PB. The Oswestry Disability Index. Spine. 2000;25(22):2940-52.

17 Saal JA, Saal JS. Nonoperative treatment of herniated lumbar intervertebral disc with radiculopathy. An outcome study. Spine. 1989; 14(4):431-7.

18 Fager CA. Observations on spontaneous recovery from intervertebral disc herniation. Surg Neurol. 1994;42(4):282-6.
19 Splendiani A, Puglielli E, De Amicis R, Barile A, Masciocchi C, Gallucci M. Spontaneous resolution of lumbar disk herniation: predictive signs for prognostic evaluation. Neuroradiology. 2004;46(11):916-22.

20 Kim ES, Oladunjoye AO, Li JA, Kim KD. Spontaneous regression of herniated lumbar discs. J Clin Neurosci. 2014;21(6):909-13.

21 Slavin KV, Raja A, Thornton J, Wagner FC Jr. Spontaneous regression of a large lumbar disc herniation: report of an illustrative case. Surg Neurol. 2001;56(5):333-7.

22 Komori H, Shinomiya K, Nakai O, Yamaura I, Takeda S, Furuya K. The natural history of herniated nucleus pulposus with radiculopathy. Spine. 1996;21(2):225-9.

23 Takada E, Takahashi M, Shimada K. Natural history of lumbar disc hernia with radicular leg pain: spontaneous MRI changes of the herniated mass and correlation with clinical outcome. J Orthop Surg. 2001;9(1):1-7.

24 Bush K, Cowan N, Katz DE, Gishen P. The natural history of sciatica associated with disc pathology. A prospective study with clinical and independent radiologic follow-up. Spine. 1992;17(10):1205-12.

25 Ahn SH, Ahn MW, Byun WM. Effect of the transligamentous extension of lumbar disc herniations on their regression and the clinical outcome of sciatica. Spine. 2000;25(4): 475-80.

26 Morgan-Hough CV, Jones PW, Eisenstein SM. Primary and revision lumbar discectomy: a 16-year review from one centre. J Bone Joint Surg. 2003;83-B:871-4.

27 Rothoerl RD, Woertgen C, Brawanski A. When should conservative treatment for lumbar disc herniation be ceased and surgery considered? Neurosurg Rev. 2002;25(3):162-5.

28 Gugliotta M, da Costa BR, Dabis E, Theiler R, Jüni $P$, Reichenbach $S$, et al. Surgical versus conservative treatment for lumbar disc herniation: a prospective cohort study. BMJ Open. 2016;6(12):e012938. 